\title{
STRATEGIES TO MINIMISE NUMERICAL VENTILATION IN CFD SIMULATIONS OF HIGH-SPEED PLANING HULLS
}

\author{
Angus Gray-Stephens \\ Department of Naval \\ Architecture, Ocean \& Marine \\ Engineering \\ The University of Strathclyde \\ Glasgow, Scotland, UK
}

\author{
Tahsin Tezdogan \\ Department of Naval \\ Architecture, Ocean \& Marine \\ Engineering \\ The University of Strathclyde \\ Glasgow, Scotland, UK
}

\author{
Sandy Day \\ Department of Naval \\ Architecture, Ocean \& Marine \\ Engineering \\ The University of Strathclyde \\ Glasgow, Scotland, UK
}

\begin{abstract}
Numerical Ventilation is a well-known problem that occurs when the Volume of Fluid method is used to model vessels with a bow that creates a small, acute entrance angle with the freesurface, typical for planing hulls and yachts. There is a general lack of discussion focusing upon Numerical Ventilation available within the public domain, which is attributable to the fact that it only affects such a niche area. The information available s difficult to find, often fleetingly mentioned in papers with a different focus. Numerical Ventilation may be considered one of the main sources of error in numerical simulations of planing hulls and as such warrants an in-depth analysis. This paper sets out to bring together the available work, as well as performing its own investigation into the problem to develop a better understanding of Numerical Ventilation and present alternate solutions. Additionally, the success and impact of different approaches is presented in an attempt to help other researchers avoid and correct for Numerical Ventilation.
\end{abstract}

Interface smearing caused by the simulations inability to track the freesurface is identified as the main source of Numerical Ventilation. This originates from the interface between the volume mesh and the prism layer mesh. This study looks into the interface to identify strategies that minimise Numerical Ventilation, presenting a novel solution to prism layer meshing that was found to have a positive impact. Through the implementation of a modified High Resolution Interface Capture (HRIC) scheme and the correct mesh refinements, it is possible to minimise the impact of Numerical Ventilation to a level that will not affect the results of a simulation and is acceptable for engineering applications.

\section{NOMENCLATURE}

Fr $=$ Froude Number

$L=$ Length Between Perpendiculars

$L_{\mathrm{tg \tau}}=$ Induced Resistance

$R_{n}=$ Reynolds Number

$R_{T}=$ Total Resistance

$R_{V}=$ Viscous Resistance

\author{
$U=$ Ship Speed \\ $x=$ Plate Length \\ $\delta=$ Boundary Layer Thickness \\ $\Delta t=$ Time Step
}

\section{INTRODUCTION}

The use of Computational Fluid Dynamics (CFD) as a tool for the hydrodynamic assessment of ships has grown considerably in the past 20 years. This is accountable to an increase in the availability of High Performance Computers and the vast speed increases this has brought about, the development of more accurate CFD codes, and the fact that users have become more confident using CFD as it has become more reliable \& established as a design tool. These factors have led to a significant increase in the associated accuracy of simulations, with statistical analysis of the 2010 Gothenburg workshop revealing that all simulations larger than $3 \mathrm{M}$ cells were within $4 \%$ of the measured resistance data, with a the mean comparison error of $-0.1 \%$, and a mean standard deviation of $2.1 \%$ [1]. With such high confidence levels in the results and its far superior post-processing abilities it is undeniable that CFD is becoming an ever more important tool in the design process of conventional ships.

Unfortunately, the same cannot be said for high-speed craft, and other non-conventional vessels. For these it is well known that resistance prediction simulations are less accurate. The ITTC noted that it is more difficult to assess the accuracy of CFD for high-speed and non-conventional vessels due to the scarcity of relevant publications [2]. Despite this, they found that for $0.3<$ $\mathrm{Fr}<0.5$ a mean prediction error of $10 \%$ is achievable. This is in line with a number of other papers published, who have reported similar levels of error.

- [3] concluded that the level of accuracy for CFD predictions is expected to be around $10 \%$

- [4] do not present their maximum and minimum errors but state that the average error was $10 \%$

- [5] found resistance errors of $4.5-9.5 \%$.

- [6] found error in the resistance predictions of 1.9 $16.7 \%$ 
- [7] were able to achieve resistance predictions with errors below $10 \%$

- [8] found an error of between $1.2-9.3 \%$. They concluded that they were able to reach comparison error values of below $7.5 \%$

The difficulties in accurately simulating high-speed craft in the fully planning condition are attributable to a number of causes. Brizzolara \& Serra reason that these difficulties in resistance prediction arise from the fact that both the pressure and viscous components are related to the dynamic lift and trim moment in a non-linear way [3]. Therefore, the accurate prediction of resistance is linked to the accurate prediction of the running trim, sinkage and hence the lift force acting upon the hull. This is expanded upon by De Luca et. al. who state that the largest errors in resistance evaluation arise from errors in the dynamic trim [8]. Their statement is based upon the observed errors in numerically calculated trim, and the relationship between dynamic trim and resistance as given by [9]:

$$
R_{T}=L_{\mathrm{tg} \tau}+R_{V}
$$

Planning hulls are typically subject to small trim angles, so a small difference in the predicted trim will lead to large variations in the wetted surface, and as such, an incorrectly calculated trim angle will affect both the pressure and viscous components resistance components.

In order to predict the trim and resistance of a hull the pressure distribution and forces acting upon the hull must be calculated accurately. To do this it is vital that a fluid of the correct properties occupies the cells adjacent to the walls. A common problem in simulations of high-speed planing hulls that prevents this from happening is that of Numerical Ventilation.

\section{NUMERICAL VENTILATION}

Numerical Ventilation (NV), or streaking, is a well-known problem when modelling planing hulls using the Volume of Fluid (VOF) model, however it is rarely mentioned or discussed in scientific papers [10]. It can be considered one of the main sources of error in numerical simulations of planing hulls [6], [8]. Böhm points out that the lack of discussion on this topic is attributable to the fact numerical ventilation only occurs with specific bodies, typically with a bow that forms a small, acute entrance angle with the free-surface as is typical for yachts and high-performance vessels [11]. There is relative scarcity of ongoing research focusing on these hullforms when compared to conventional vessels, for which this problem does not occur. As such, there is limited discussion upon NV.

NV occurs when the free-surface interface is not properly captured. Particles of air become trapped in the boundary layer in the first few cells nearest the wall and are transported under the hull. Olin reasons that this is introduced in the forward most spray area due to the fact that at some point along the hull, where the spray thickness approaches zero, the local cell size will be the same order of magnitude as the spray thickness. The refinement in this area will not be sufficient to resolve the spray sheet, and as such no spray sheet will form forward of the stagnation line. As opposed to forming a spray sheet, the information in these cells will be supplied under the hull and cause NV [12].

Another presented explanation focuses upon the interface capturing method that is employed by the simulation. Star $\mathrm{CCM}+$ uses the well-known High Resolution Interface Capturing (HRIC) scheme. This is based upon the Compressive Interface Capturing Scheme for Arbitrary Meshes and was introduced by [13] and developed by [14]. The standard HRIC scheme is modified based on the local Courant Friedrichs Lewy (CFL) number so that it blends the HRIC scheme and the Upwind Differencing (UD) scheme [8]. However it has been found that utilising a local CFL dependent scheme spreads the freesurface [15] which [8] presents as the potential main cause of NV.

If $\mathrm{NV}$ occurs, it has a notable effect on the calculation of the vessels frictional resistance [10], [12]. The CFD code will compute a lower value for the shear stress as this component is calculated using the velocity and viscosity of the elements in the boundary layer. If $\mathrm{NV}$ has occurred this cell may contain a mixed fluid, and thus the properties of this mixed fluid (which has a lower viscosity) will be used as opposed to those of water. [10] presents a detailed examination of this effect. It will also have an impact on the calculation of the pressure distribution and the trim of the hull, which is known to have a large impact on both the frictional and viscous resistance components of a planning hull due to the small trim angles at which they operate.

A number of strategies to minimize the problem of numerical ventilation have been proposed:

1. Viola et. al. found that using first order discretization for the convection terms lead to an increase in NV [10]. It also found that using first order discretization lead to an increase in numerical diffusion \& an increase in the computed resistance. The interface between water and air became less sharp and the transition occurred over a greater number of cells.

2. Viola et. al. also found that the timestep had an impact on whether NV was present or not [10]. As the timestep was increased so did the effects of NV.

3. Viola et. al. proposed a method to artificially suppress NV [10]. A source term is included in the transport equation for the air phase for the cells adjacent to the wall boundary layer. This removes the air mass from affected cells and replaces it with water. De Luca et. al. 
note that this strategy may introduce errors in the conservation properties of mass and momentum [8], however [10] states that despite the violation of the continuity equation the effect on results is negligible.

4. Olin found that it was possible to reduce, but not suppress the NV through mesh refinement. The author states that a refined mesh close to the hull is not sufficient to reduce the effect, however a refinement upstream of the hull along the water surface has a positive impact [12].

5. Böhm reasons that as simulations of towing tank procedures seek a steady state solution, the robustness of the HIRC scheme that is modified with respect to the local CFL number is not required. As such, it is possible to modify this scheme to remove the switch that blends it with the UD scheme as it is known that a UD scheme leads to interface smearing [15]. Böhm found that this approach was well suited and gave a much sharper freesurface interface, resulting in the minimization of numerical ventilation [11], as did De Luca et. al. [8]. It was also found that this approach had a positive impact on the calculated wave patterns due to the fact there was less interface smearing.

Böhm has performed the most extensive work on NV, comparing artificial suppression as suggested by [10] with his modified HRIC scheme and a standard HRIC scheme [11]. He found artificial suppression to be the most successful at removing $\mathrm{NV}$, however advised caution as it introduces errors into the conservation properties of mass and momentum. It was also found that whilst not as impressive as artificial suppression, the modified HRIC scheme was far superior to the standard HRIC scheme.

Whilst the artificial suppression method is the most successful at eradicating $\mathrm{NV}$, it should be noted that it is not always possible to utilise this approach. This is especially true when working with hulls for which air is purposefully introduced to the flow such as stepped-hulls or air-lubricated hulls. In these cases, artificial suppression would be unable to differentiate between air accountable to $\mathrm{NV}$ and air that has been purposefully introduced. For these cases other methods must be investigated and a deeper understanding of the causation of NV developed.

\section{PROJECT AIMS}

It is apparent that NV is a wide spread problem for simulations of planning hulls. There are a range of methods to minimise the effects, however there is no definitive solution to the problem and little work in the public domain discussing it. This paper sets out to bring together the available work, as well as performing its own investigation into the problem to develop the understanding of $\mathrm{NV}$ and present alternate solutions.
Additionally, the success and impact of different approaches is presented in an attempt to help other researchers avoid NV.

\section{METHEDOLOGY}

The study will use the published calm water experimental results of a series of high-speed hard chine planing hulls, generated by Taunton et. al. at Southampton University. For details on how these were generated please refer to [16]. Model $\mathrm{C}$ was selected at a speed of $9.21 \mathrm{~m} / \mathrm{s}$ as a benchmark case as it was in line with the upper Froude numbers of similar studies investigating planing hulls through CFD.

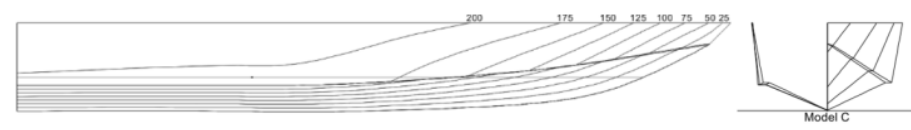

FIGURE 1 - LINES PLAN OF MODEL C

Simulations will be set up using CD Adapco's Star CCM+ CFD solver and run on the ARCHIE-WeST High Performance Computer, hosted by the University of Strathclyde.

\section{NUMERICAL MODELING}

This section will provide details upon the numerical simulation approaches utilised by this study, however it will not provide detailed information upon the numerical workings of the CFD code. Detailed information into the inner workings of CFD can be found in [17].

\section{Physics Modelling}

Larson et. al. state that the two-equation turbulence models have been shown to give accurate predictions in ship hydrodynamics [18]. The ITTC concluded from their analysis of the entries to Gothenburg 2010 Workshop that there was no visible improvement in accuracy for resistance prediction when turbulence models that are more advanced than the twoequation models were used [1]. It found that $k-\omega$ was by far the most applied turbulence model with $80 \%$ of the submissions for the workshop using some form of variation of it. The ITTC also concluded that for resistance calculations the turbulence modelling has little effect on the prediction accuracy [2].

A review of other studies using CFD for planing hull performance prediction found that the majority of simulations use either $k-\varepsilon$ [8], [19]-[23] or $k-\omega S S T$ [5]-[7], [24][26]. Whilst both models have been shown to be comparable in terms of resistance prediction the $k-\omega S S T$ is known to be superior at predicting separating flows and wake patterns [1], [2]. As such, this model was selected despite the fact that it is more computationally expensive.

The Volume of Fluid (VOF) method was used to model and track the position of the freesurface. This simple-multiphase 
model is well-suited for simulating flows of immiscible fluids and is known for its numerical efficiency. When the VOF model is used a new variable is introduced to define the spatial distribution of each phase at a given time. This is known as the volume fraction. A volume fraction of 0.5 represents a cell that contains $50 \%$ water and $50 \%$ air, and as such this is used to define the freesurface. To help ensure that there was a sharp interface between the phases a second order spatial discretization scheme was used, as suggested by [27].

An all wall $\mathrm{y}+$ wall treatment was selected. This is a hybrid approach that emulates a low $\mathrm{y}+$ wall treatment for fine meshes $(y+>1)$ and the high $y+$ wall treatment for course meshes $(\mathrm{y}+<30)$. It is capable of producing reasonable answers for meshes of intermediate resolution $(1<y+<30)$ through the use of a blending function.

An average $y^{+}$of 40 was achieved on the wetted hull. This meant that for the wetted surface the viscous sublayer was not resolved and instead wall functions are used to obtain the boundary conditions of the continuum equations. The main advantage of the high $\mathrm{y}+$ wall treatment is that there are significant savings in computational time due to the reduction in the number of near-wall cells [27].

A second order convective discretization scheme was chosen in line the findings of Viola et. al. to minimise NV and improve the accuracy of the simulation [10].

\section{Timestep}

The timestep can be selected either to satisfy the CFL condition or to resolve the flow features of interest. The ITTC recommend that for standard pseudo-transient resistance simulations a timestep that will satisfactorily resolve the flow features is a function of the vessels speed and the length of the hull, such that [28]:

$$
\Delta t=0.005 \sim 0.01 \frac{L}{U}
$$

An extensive timestep study found that satisfying the CFL condition for all cells resulted in an unjustifiable increase in computational time with a negligible impact upon the results. It was also determined that a timestep that was coarser than the ITTC recommendations was suitably accurate, and that there was an insignificant impact upon the results. As such the following timestep was selected.

$$
\Delta t=0.02 \frac{L}{U}
$$

The ITTC define $\mathrm{L}$ as the length between perpendiculars of the vessel. For the purpose of this study, $\mathrm{L}$ in the timestep calculation was taken to be the wetted length of the keel of the vessel.

\section{Computational Domain}

It is well known that when using CFD the domain must be an appropriate size, with boundaries being placed sufficiently far from the hull to ensure they have no effect on the solution. The ITTC recommend that the inlet and exterior boundaries are located 1-2 L from the hull, with the outlet being placed 3-5 L downstream [18]. Care was taken to ensure that the wake of the hull would not intersect with the exterior boundary. The size of the computational domain was selected in accordance with the ITTC recommendations [18] and can be seen in Figure 2

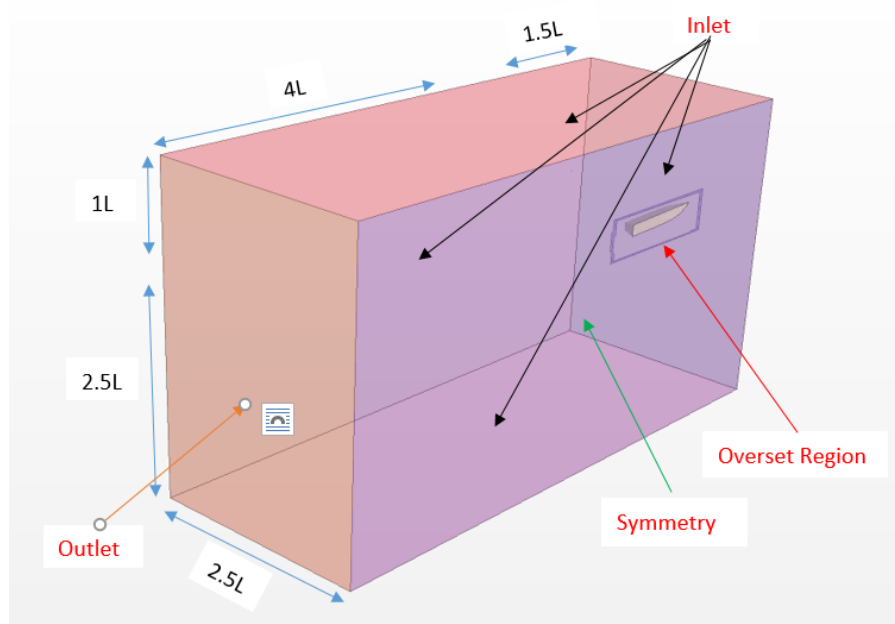

FIGURE 2 - BOUNDARY CONDITIONS AND DOMAIN SIZES

The VOF Wave Damping option was utilised to apply a damping zone of $2.5 \mathrm{~m}$ to the side and outlet in order to reduce wave reflections and the influence of the boundaries on the solution. This damping introduces a vertical resistance to vertical motion and suppresses waves.

A preliminary simulation was run with wave damping included and excluded. It was found to have minimal effects on the results $(-0.016 \%$ error in resistance, $0.048 \%$ in trim and $0.012 \%$ in sinkage), which is confirmation that the boundaries were placed far enough from the hull for wave reflection to have minimal influence. Interestingly, it was found that the inclusion of wave damping reduced the runtime of the simulation by $1.77 \%$.

\section{Boundary Conditions}

In all CFD simulations, the selection of appropriate boundary conditions is vital for both the determination of an accurate solution and the prevention of unnecessary computational costs. As is common practice in marine resistance simulations in all cases only half of the hull was modelled, with a symmetry condition being used at the centerplane of the domain to ensure 
the flow predictions remained accurate. This halves the computational demand over modelling the whole hull.

A study was carried out to determine the effects on both accuracy and run time for the various combinations of boundary conditions. It should be noted that the boundary condition study was done using one of the preliminary meshes used to establish the final set up used for this study, however it remained constant for the duration of the BC study.

1. Non-Slip walls - Non-slip walls used for bottom and side, Inlet used for top

2. Symmetry - Symmetry Plane used to top, bottom and side

3. Inlet \& Symmetry - Inlet used for top and bottom, Symmetry Plane used for side

4. Slip walls - Slip walls used for bottom and side, Inlet used for top

5. Inlet- Inlet used for top, side and bottom

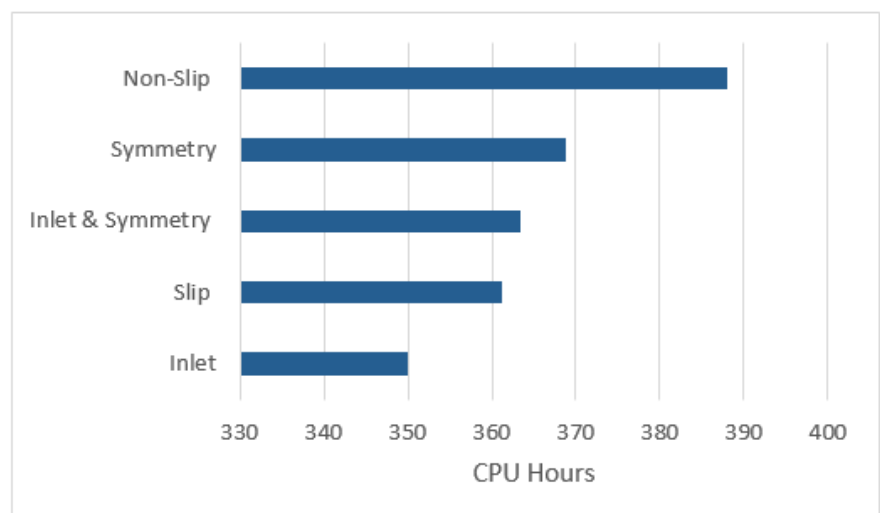

FIGURE 3 - RESULTS OF THE BC STUDY

In all cases aside the non-slip one there was a $0.041 \%$ variation in resistance error, $0.078 \%$ in trim, and $0.070 \%$ for sinkage. The non-slip case had a resistance error of $0.8 \%$ less, a trim error of $7.5 \%$ less and a sinkage error of $10.6 \%$ more.

The inlet conditions were selected as they gave the fastest flow solutions, with little to no effect on the accuracy of the simulation. The non-slip condition was not selected despite showing to be the most accurate as it was the least physically representative of the problem being modelled. Given the depth of the tank and the draft of the vessel, the problem is considered a deep-water problem and as such, the presence of the bottom of the tank should have negligible impact upon the results.

\section{Computational Grid}

The Dynamic Fluid Body Interaction (DFBI) module allows a simulation to include the motion of a vessel in response to the shear and pressure forces exerted by the flow, and to any additional forces that are user defined. Star $\mathrm{CCM}+$ calculates the force and moments that act upon the vessel before solving the governing equations of rigid body motion to determine the new position of the vessel. This model allows a body to have up to six degrees of motion, however to simplify the simulation the vessel will only be free to move in two - pitch and heave. The equilibrium motion option is employed to achieve a quasisteady-state equilibrium position of the vessel. This option means the body motion is calculated between longer intervals to reduce the time required to achieve a steady position [27].

The hydrodynamic field generated by a planning hull is far more complex than that of a conventional displacement hull with a small error in the predicted trim having a large impact upon the total resistance. There are a number of approaches that may be considered non-conventional for calm water resistance simulations that allow the mesh to change dynamically with the motion of the hull when implemented. These approaches help simulations maintain numerical accuracy while the hull is in inclined positions [29].

The most complex and computationally demanding of the approaches is the Chrimea Grid, or Overset Mesh. Overset Meshes typically involve a background mesh that is tailored to the environment, and one or more overset grids that are tailored and attached to the body, which overlaps with the background mesh. An overset mesh approach is very useful when dealing with moving bodies and Fluid Structure Interaction (FSI) as it offers far greater flexibility over standard meshing techniques. The approach's key advantage is that the grid system around the hull moves with the hulls motion. This means that the remeshing or deformation of elements is not required, and the mesh remains consistent in terms of element quality. It is well known to be capable of modelling the large motions of a planing hull, and is recommended for configurations involving body-motion [18]. As such it was decided to implement an overset mesh approach.

The mesh was generated using the automated meshing capability of Star $\mathrm{CCM}+$, which relies upon the Cartesian cutcell method. The trimmed cell mesher presents a robust and efficient method of producing a high-quality grid, predominantly made up of unstructured hexahedral cells with polyhedral cells next to the surface. It constructs a template mesh from the target sizes and then trims this using the input surfaces. It allows for a large degree of control through the use of local surface and volumetric controls that allow the user to increase or decrease the mesh density. Growth parameters can also be used to ensure that there is a smooth transitioning of the mesh and prevent the introduction of numerical errors.

The mesh was set up with areas of progressively refined mesh to ensure each area of interest was sufficiently fine. Three layers of refinement were used for the freesurface, the hull box and the wake region. Additional refinements were included for the bow, the stern and for the freesurface upstream of the hull, as will be discussed later. The refinements can be seen in Figure 4. Care was taken to follow the overset guidelines as laid out by [27]. 
Of key importance was to ensure that cells in the overlapping region between the overset and background meshes are of similar sizes. This helps reduce any interpolation errors to be of the same order as other discretization errors.

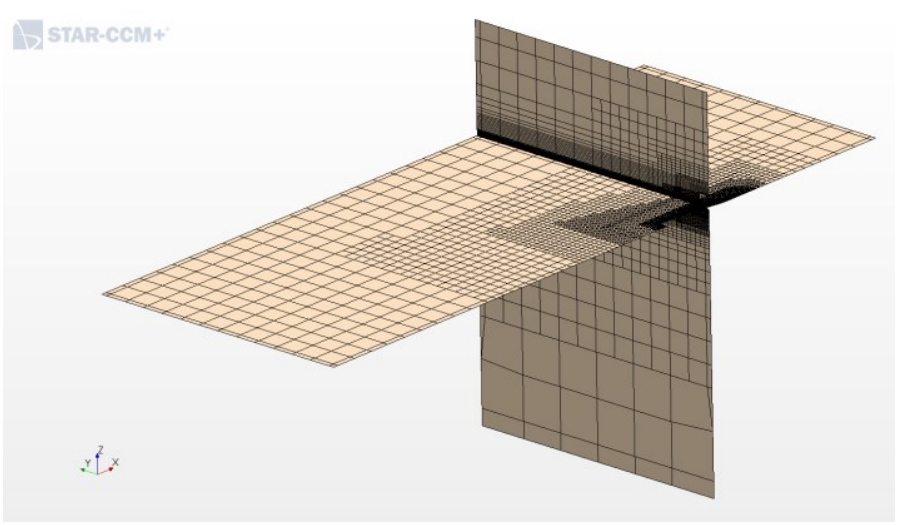

FIGURE 4 - COMPUTATIONAL GRID

The prism layer mesher was used in conjunction with the trimmed cell mesher to generate orthogonal prismatic cells next to the hull. Utilising the prism layer mesher generates highaspect ratio cells that are aligned with the flow next to the wall. This allows the software to resolve high velocity gradients that are associated with the boundary layer and increases the accuracy of the simulation. The initial thickness of the prism layer was calculated as the thickness of the turbulent flow over a flat plate, as given by:

$$
\frac{\delta}{x}=0.37 R_{n}^{-\frac{1}{5}}
$$

A stretching ratio of 1.2 as suggested by [18] was utilised, with a first wall cell height that was calculated to give a $\mathrm{y}+$ of 40 . The thickness of each layer of the cells in the prism layer was calculated and the layer of a size that would naturally grow into the cell size of the volume mesh was chosen as the final prism layer. Whilst this meant that the prism layer thickness was $0.015 \mathrm{~m}$ as opposed to $0.020 \mathrm{~m}$ it ensured there was a far smother transition in the mesh. Without this alteration the outer prism layer cells would have been larger than the volume mesh beside them.

For the purpose of investigating numerical ventilation a number of meshes were used ranging from $2.5 \mathrm{~m}-6 \mathrm{~m}$ cells. The final mesh that was developed and will be used for the continuation of this work contained $6 \mathrm{~m}$ cells.

\section{RESULTS \& DISCUSSION}

Following the initial run of the simulation, it was confirmed that it was subject to a major NV problem. Through the course of the study the problem of NV was minimised from a level at which it had a large negative impact upon the calculated results, to a level at that was acceptable for engineering applications and had a minimal effect on the results. The progression can be seen in Figure 5-8 Through a combination of the modified HRIC scheme and the correct mesh refinement, it was possible to minimise NV to two thin streaks containing $96-98 \%$ water.

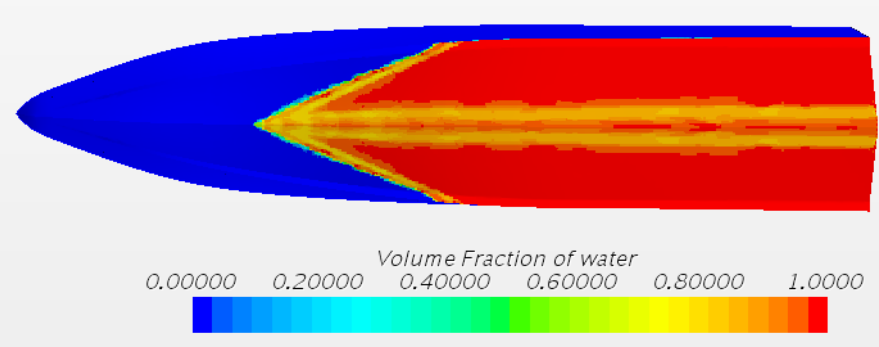

FIGURE 5 - SIMULATION WITH STANDARD HRIC SCHEME

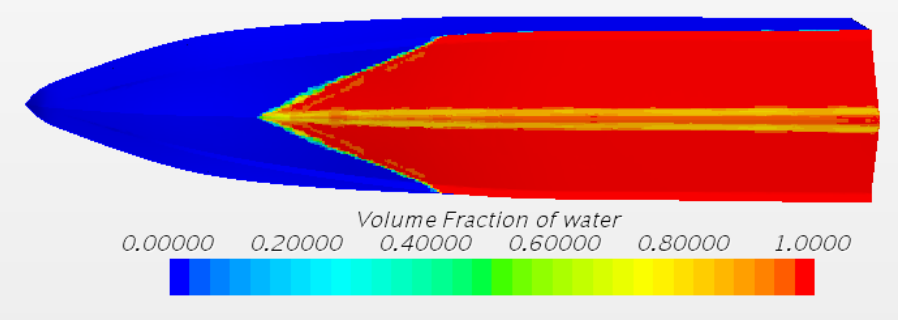

FIGURE 6 - SIMULATION WITH MODIFIED HRIC SCHEME

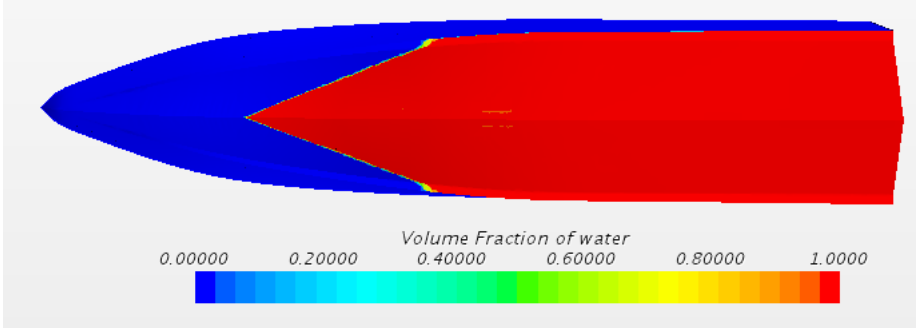

FIGURE 7 - SIMULATION WITH MODIFIED HRIC SCHEME \& MESH REFINEMENT

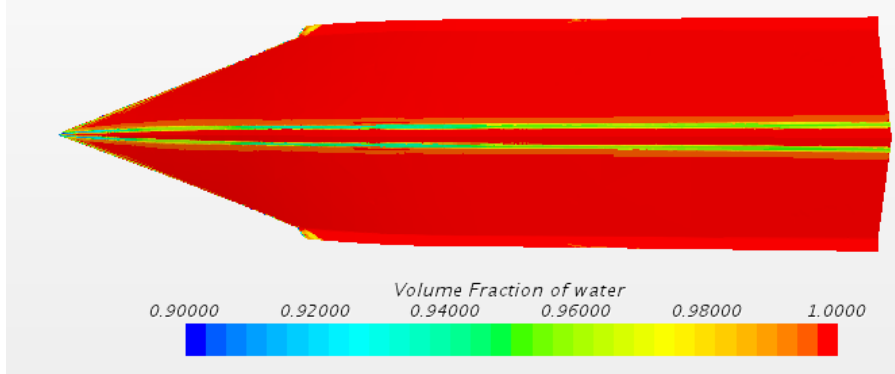

FIGURE 8 - SIMULATION WITH MODIFIED HRIC SCHEME \& MESH REFINEMENT ZOOMED IN 
The following section will discuss the potential solutions that were tested and their degree of success, as well as discussing the root cause of NV.

\section{Modified HRIC Scheme}

As was suggested by Böhm, and utilised by several other authors investigating high speed planing hulls through the use of CFD a modified HRIC scheme was employed [11]. Due to the removal of the CFL dependency and the blending with an UD scheme the ability of the simulation to capture the interface between the two phases was improved. This led to a significant reduction in the $\mathrm{NV}$ as well as giving the remaining $\mathrm{NV}$ a far sharper interface.

\section{Lowering CFL Number}

The CFL number is the ratio of the time-step to the mesh convection time-scale. It essentially defines the number of cells that a particle of fluid will pass through in each timestep. It is recommended that the CFL number is less than or equal to 1 for numerical stability, however as a calm water resistance simulation is seeking a steady state solution larger CFL numbers give equally accurate results. It was theorized that a large CFL number at the point of hull entry would result in the code "losing track" of the air partials and introduce them into the flow under the hull due to the fact they were travelling through multiple cells in every timestep. A range of CFL numbers were tested and it was found to have little to no effect on NV. In the timestep study the smallest timestep had a CFL of around 12 at the point of hull entry, whilst the largest timestep had a CFL of around 100. There was no noticeable effect on the NV between the two.

As a final check the CFL was lowered to have a value of 0.5 at the point of hull entry, which required a timestep 20 times smaller than the ITTC formulation. This was found to reduce the percentage of air in the streaks from $3.5 \%$ to $2 \%$, a small improvement, however it did not justify the extra computational time.

\section{Boundary Conditions}

As part of the BC study the NV was also checked. It was found that the choice of BC's had no impact upon the NV that was present.

\section{Domain Size}

A smaller domain of the same dimensions as the towing tank in which the tests were originally carried out was also tested. The domain size was also found to have no impact upon the NV.

\section{Turbulence Model}

The $k-\varepsilon$ turbulence model was tested, however it was found to have no impact upon the NV.

\section{Sharpening Factor}

The sharpening factor attempts to reduce numerical diffusion and improves the resolution of the interface between phases. It does this by introducing a new anti-diffusion velocity term into the VOF transport equation. A known problem with increasing the sharpening factor is that it may result in a non-physical alignment of the freesurface with the gridlines, which was found to result in a much flatter wake. A sharpening factor of 0.2 was included but it was found to be detrimental. Rather than sharpening the interface and preventing $\mathrm{NV}$ it was found to sharpen the interface of the NV under the hull, resulting in more clearly defined streaking.

\section{Mesh Refinement}

Mesh refinement was the best solution to the NV problem, after the modified HRIC scheme. The root cause of NV is when the freesurface interface becomes blurred. The modified HRIC Scheme helps prevent this, which is accountable to its success. An inadequate mesh may also result in interface smearing through a number of causes.

The first cause of interface smearing accountable to the mesh arises from the prism layer. As was discussed earlier NV only occurs for specific bodies, typically with a bow that forms a small, acute entrance angle with the freesurface. When bodies such as these are meshed the prism layer mesh and the volume mesh that have a small angle between them, as seen in Figure 9. In the case of a conventional ship this angle would be large, possibly even 90 degrees. Due to this the cells in the prism layer mesh are not aligned with the flow. It is well known that when the freesurface is not aligned with the mesh numerical diffusion will occur. This results in interface smearing at the point of entry of the hull, as seen in Figure 9. It can also be seen that air is transported under the hull in the near wall cells, resulting in NV.

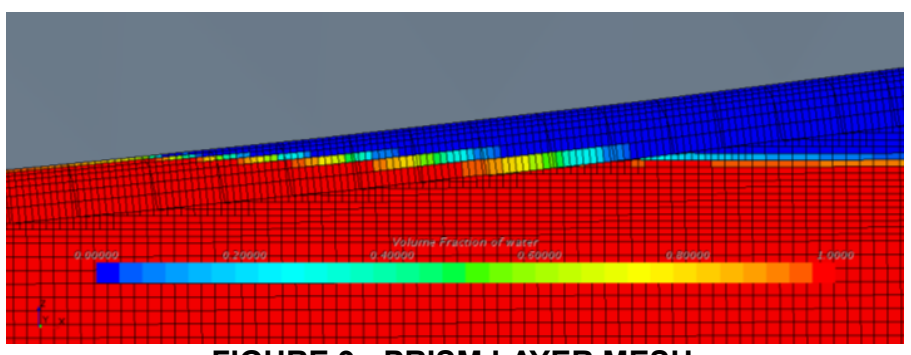

FIGURE 9 - PRISM LAYER MESH

Any meshing strategy that prevented interface smearing in the prism layer resulted in a vast improvement on NV. The number of prism layers had a large effect. It is normally advisable to have the last cell of the prism layer and the first cell of volume mesh of comparable sizes, however implementing this was found to be detrimental. As can be seen in Figure 9 the last layer of the prism layer appears to be too large, however adding more prism layers was found to result in further interface smearing. During the preliminary set up of the simulation a low $\mathrm{y}+$ wall treatment was initially attempted, but it was found that the large 
number of prism layers resulted in far more interface smearing, as seen in Figure 10. It should be noted that a modified HRIC has not been implemented in Figure 10, however the smearing was considerably worse when compared with the same simulation with a high $\mathrm{y}+$ wall treatment, the only difference being the number of prism layers. This finding reinforces the idea that reducing the number of prism layers plays a large part in preventing NV.

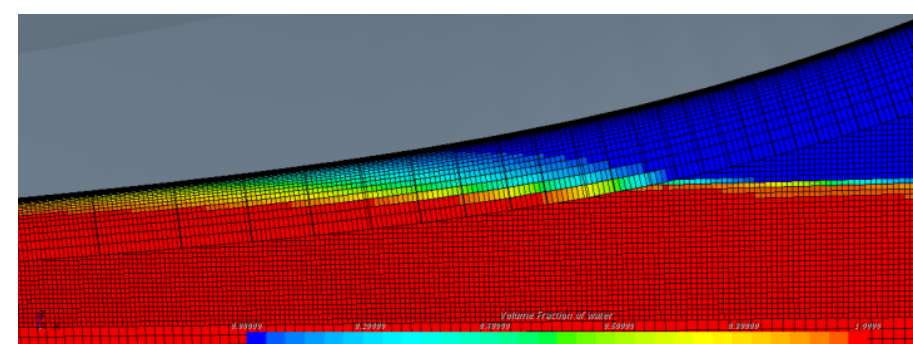

FIGURE 10 - LOW Y+ WALL TREATMENT INTERFACE SMEARING

The thickness of the prism layer also had a large effect on NV. A thick prism layer meant that the freesurface had a considerably larger zone in which interface smearing occurred as there were more cells that were misaligned with the flow. A strategy to minimise this was developed, in which the prism layer thickness was reduced at the point of hull entry, as seen in Figure 11.

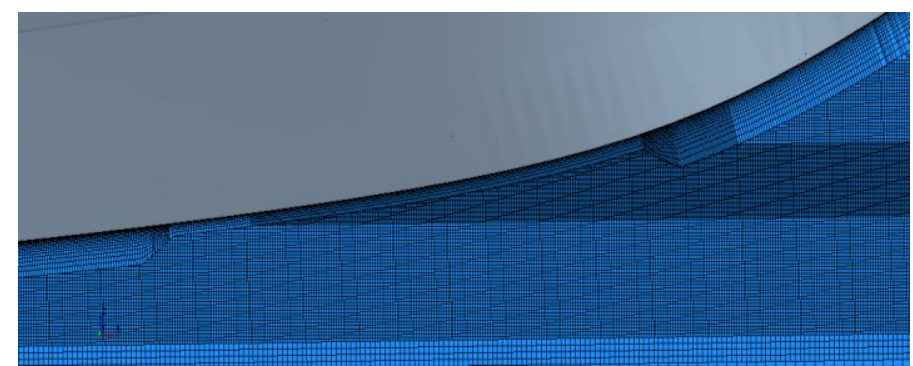

FIGURE 11 - COLLAPSING PRISM LAYER

The second cause of freesurface blurring arose from inadequate mesh refinements. The first refinement strategy was to increase the resolution of the mesh in the bow area. This ensures that the mesh is capable of resolving the thin spray root that forms and prevents air being dragged under the hull. The bow refinement on its own however, was found to be detrimental to the NV. This was because the bow refinement had a finer z-refinement than the freesurface refinement, which resulted in the interface smearing when the freesurface entered the bow-refinement zone; the freesurface that was modelled by one cell was modelled by multiple cells, as seen in Figure 12. It is therefore necessary to also include an upstream freesurface refinement zone of equal z-refinement to that of the bow-refinement. This is in agreement with what was found by Olin, who stated that upstream refinement had the largest positive impact [12].

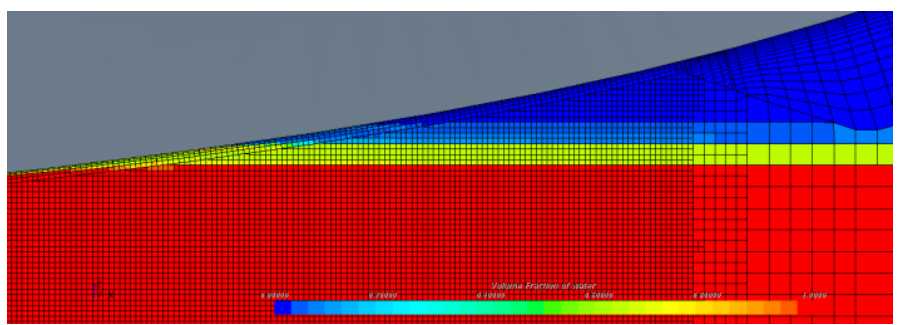

FIGURE 12 - INTERFACE SMEARING DUE TO DOWNSTREAM Z-REFINEMENT

Adding upstream freesurface refinement had a larger effect than the bow refinement. This is accountable to the way in which Star CCM + generates the mesh. When an upstream refinement added, the refinement is projected through the prism layer mesh onto the hull surface mesh as well in the zone where the upstream refinement meets the hull. This means that when a freesurface refinement is implemented it essentially adds a surface refinement to the hull in the area in which it intersects with the hull. Adding a bow refinement in addition to the upstream refinement helps ensure that the refined area is sufficiently large to capture the flow characteristics in the entry region. Having a bow refinement was found to further minimise $\mathrm{NV}$ over an upstream freesurface refinement alone.

When the levels of refinement were varied for each of the two zones it was found to have a large effect on the NV. The level of bow refinement had less impact as the freesurface refinement projects onto the hull at the intersection zone, but it still showed some effect. The level of freesurface refinement had a notable effect on the NV. There appears to be a 'sweet spot' for the level of both refinements that was found by making systematic variations. If they are more or less refined than this 'sweet spot' then the NV becomes worse. Of interest is that this 'sweet spot' is relative to the rest of the mesh, rather than absolute sizes. This was noted during a mesh study when the sizing of the entire mesh was altered but the level of NV remained constant despite the fact that the absolute size within the bow and upstream refinements had changed. Previously changing the absolute sizes in these zones and leaving the mesh constant had resulting in increased $\mathrm{NV}$.

Whilst altering the mesh a second source of NV was discovered further aft of the bow entry point. This resulted in two additional streaks as seen in Figure 13.

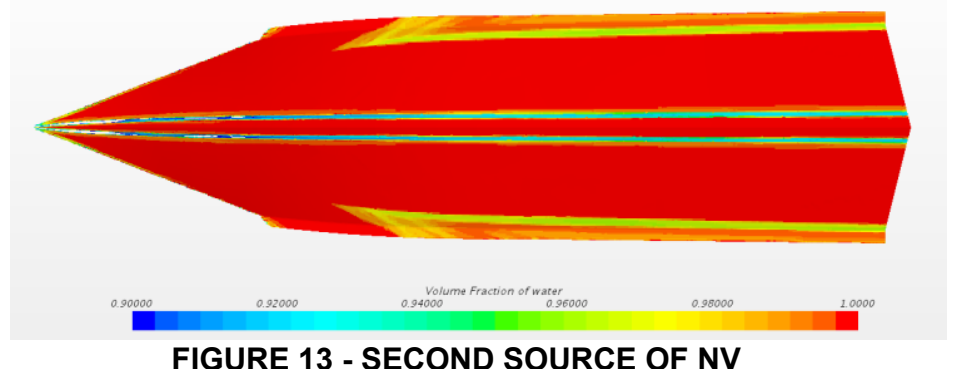

FIGURE 13 - SECOND SOURCE OF NV 
This was once again the result of interface smearing where the volume mesh met the prism layer. In this case, the volume mesh cells were considerably larger than the prism layer, which lead to smearing as seen in Figure 14. As the slice used to generate the scene was systematically moved aft the interface smearing could be seen as it was developed into a bubble that was drawn into the prism layer. Here it moved inwards until it smeared on the hull. The solution to this second source was to ensure that the volume and prism layer mesh were of comparable sizes until the freesurface met the chine. In a practical sense, this was resolved by extending the upstream freesurface refinement aft until it met the chine.

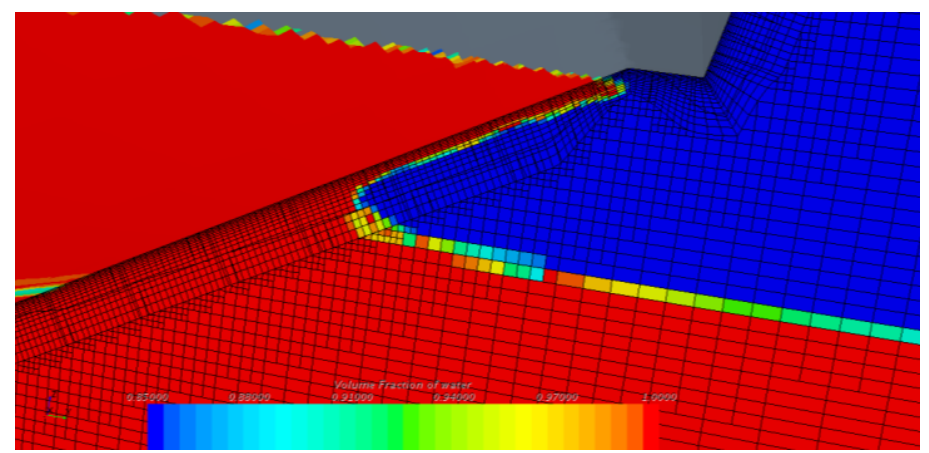

FIGURE 14 - INTERFACE SMEARING AT SECOND SOURCE OF NV

\section{CONCLUSION}

The main cause of NV identified in this study is when interface smearing occurs due to the simulation being incapable of tracking the freesurface. This may be accountable to either the interface capturing scheme or the mesh. When the mesh was at fault, NV was introduced to the hull from two different sources:

- at the point of entry

- as streaks nearer the chine

When a more detailed understanding of the problem was developed and both sources were investigated, it was found that they both originated from the same cause - the interface between the prism layer mesh and the volume mesh. This is primarily accountable to the fact that the prism layer cells are not aligned with the freesurface, which results in numerical diffusion. Whilst there is no way to avoid this a number of solutions were tested. It was found that whilst it is not possible to eradicate $\mathrm{NV}$ it is possible to reduce it to a level at which it will have little to no bearing on the results and is acceptable for engineering applications. Through the course of this study the NV present in the simulation was reduced from two $0.055 \mathrm{~m}$ wide streaks with $90 \%$ air content, to two $0.011 \mathrm{~m}$ wide streaks of $4 \%$ to $2 \%$ air.

Previous work that presented solutions to NV was compiled and the applicability of each was tested. It was found that Bohm's modified HRIC [11] and Olin's upstream refinements [12] were the most capable, however from the literature it is suggested to use Viola et. Al's. artificial suppression where applicable [10]. It was found that the timestep and the CFL of the freesurface had little impact when trying to reduce NV. Additionally, a number of other solutions were tried to help gain a better understanding of NV and to help future researchers save time by establishing what is and is not successful.

A novel solution of reducing the thickness of the prism layer at the point of water entry was developed and deemed successful. Through the investigation into the effects of mesh parameters on NV and by laying out clearly how interface smearing may be introduced through an inadequate mesh it is hoped that the understanding of the $\mathrm{NV}$ will be further developed. A more detailed and widespread understanding of NV will hopefully increase the accuracy of high-speed planing hull simulations and by minimising the problem it will ensure that all future work is more reliable. Future work in the area should investigate the interface capturing scheme settings in more detail. Whilst it is possible to minimise Numerical Ventilation through the mesh refinement it may not be possible to eradicate it fully using this approach. 


\section{REFERENCES}

[1] L. Larson, F. Stern, and M. Visonneau, Numerical Ship Hydrodynamics - An Assesment of the Gothenburg 2010 Workshop. 2014.

[2] ITTC Specialist Committee on CFD in Marine Hydrodynamics, "Specialist Committee on CFD in Marine Hydrodynamics Final report and Recommendations to the 27 th ITTC," 2014.

[3] S. Brizzolara and F. Serra, "Accuracy of CFD codes in the prediction of planing surfaces hydrodyamic characteristics," 2nd Int. Conf. Mar. Res. Transp., no. June 2007, pp. 147-158, 2007.

[4] S. T. G. Veysi, M. Bakhtiari, H. Ghassemi, and M. Ghiasi, "Toward numerical modeling of the stepped and non-stepped planing hull," J. Brazilian Soc. Mech. Sci. Eng., vol. 37, no. 6, pp. 1635-1645, 2015.

[5] S. Mancini, F. De Luca, and A. Ramolini, "Towards CFD guidelines for planing hull simulations based on the Naples Systematic Series," in 7th International Conference on Computational Methods in Marine Engineering, MARINE 2017, 2017, vol. 2017-May, no. May.

[6] A. De Marco, S. Mancini, S. Miranda, R. Scognamiglio, and L. Vitiello, "Experimental and numerical hydrodynamic analysis of a stepped planing hull," Appl. Ocean Res., vol. 64, no. March, pp. 135154, 2017.

[7] D. Frisk and L. Tegehall, "Prediction of High-Speed Planing Hull Resistance and Running Attitude. A Numerical Study Using Computational Fluid Dynamics," Gothenburg, 2015.

[8] F. De Luca, S. Mancini, S. Miranda, and C. Pensa, "An Extended Verification and Validation Study of CFD Simulations for Planing Hulls," J. Sh. Res., vol. 60, no. 2, pp. 101-118, 2016.

[9] W. Sottorf, "Experiments with planing surfaces," Natl. Advis. Comm. Aeronaut., Mar. 1932.

[10] I. M. Viola, R. G. J. Flay, and R. Ponzini, "CFD analysis of the hydrodynamic performance of two candidate America's cup AC33 hulls," Int. J. Small Cr. Technol., vol. 154, no. 1, pp. 1-12, 2012.

[11] C. BÖHM, "A Velocity Prediction Procedure for Sailing Yachts With a Hydrodynamicmodel Based on Integrated Fully Coupled Ranse-Free-Surface Simulations," Delft University of Technology, 2011.

[12] Linus Olin, "Numerical modeling of spray sheet deflection on planing hulls," KTH Royal Institute of Technology, Stockholm, 2015.

[13] O. Ubbink, "Numerical prediction of two fluid systems with sharp interfaces," University of London and Diploma of Imperial College, 1997.

[14] S. MUZAFERIJA and M. PERIC, "Computation of free surface flows using interface-tracking and interface capturing methods," in Nonlinear Water Wave Interaction, Southampton: WIT Press, 1999.

[15] Y. Andrillon and B. Alessandrini, "A 2D+T VOF Fully Coupled Formulation for Calculation of Breaking Free Surface Flow," in Twenty-Fourth Symposium on Naval Hydrodynamics, Washington, D.C.: National Academies Press, 2003.

[16] D. J. Taunton, D. A. Hudson, and R. A. Shenoi, "Characteristics of a series of high speed hard chine planing hulls - Part 1: Performance in calm water," Int. J. Small Cr. Technol., vol. 152, pp. 55-75, 2010.

[17] J. H. Ferziger and M. Perić, Computational Methods for Fluid Dynamics, Thrid. Berlin: Springer, 2002.

[18] ITTC - Recommended Procedures and Guidelines, "Practical Guidelines for Ship CFD," 2011.

[19] S. Brizzolara and D. Villa, "CFD SIMULATION OF PLANING HULLS," in Seventh International Conference On High-Performance Marine Vehicles, 2010.

[20] P. Lotfi, M. Ashrafizaadeh, and R. K. Esfahan, "Numerical investigation of a stepped planing hull in calm water," Ocean Eng., vol. 94, pp. 103-110, 2015.

[21] M. Bakhtiari, S. Veysi, and H. Ghassemi, "Numerical Modeling of the Stepped Planing Hull in Calm Water," Int. J. Eng., vol. 29, no. 2, Feb. 2016.

[22] O. F. Sukas, O. K. Kinaci, F. Cakici, and M. K. Gokce, "Hydrodynamic assessment of planing hulls using overset grids," Appl. Ocean Res., vol. 65, pp. 35-46, 2017.

[23] A. Dashtimanesh, A. Esfandiari, and S. Mancini, "Performance Prediction of Two-Stepped Planing Hulls Using Morphing Mesh Approach," J. Sh. Prod. Des., vol. 00, no. 0, pp. 1-13, 2017.

[24] H. Ghassemi, M. Kamarlouei, and S. T. G. Veysi, "A Hydrodynamic Methodology and CFD Analysis for Performance Prediction of Stepped Planing Hulls," Polish Marit. Res., vol. 22, no. 2, pp. 23-31, 2015.

[25] S. Wang, Y. Su, X. Zhang, and J. Yang, "RANSE simulation of high-speed planning craft in regular waves," J. Mar. Sci. Appl., vol. 11, no. 4, pp. 447-452, 2012.

[26] T. Castiglione, F. Stern, S. Bova, and M. Kandasamy, "Numerical investigation of the seakeeping behavior of a catamaran advancing in regular head waves," Ocean Eng., vol. 38, no. 16, pp. 1806-1822, 2011.

[27] CD Adapco, "Simcenter STAR-CCM+ Documentation." 2018.

[28] 26th ITTC Specialist Committee on CFD in Marine Hydrodynamics, "Practical Guidelines for Ship CFD Applications," 2014.

[29] E. Begovic, C. Bertorello, and S. Mancini, "Hydrodynamic performances of small size swath craft," Brodogradnja/Shipbuilding, vol. 66, no. 4, pp. 1-22, 2015. 